\title{
Parallel Machine Problems with Equal Processing Times: A Survey
}

\author{
Svetlana A. Kravchenko • Frank Werner
}

\begin{abstract}
The basic scheduling problem we are dealing with is the following. There are $n$ jobs, each requiring an identical execution time. All jobs have to be processed on a set of parallel machines. Preemptions can be either allowed or forbidden. The aim is to construct a feasible schedule such that a given criterion is minimized. For a couple of problems of this type, recently the complexity status has been solved and several interesting results have been presented. In this paper, we survey existing approaches for the problem class considered.
\end{abstract}

\section{Introduction}

We consider the following basic scheduling problem. There are $n$ jobs, denoted by $J_{1}, J_{2}, \ldots, J_{n}$, each requiring an identical execution time $p$. With each job $J_{j}, j=$ $1, \ldots, n$, there can be associated a release time $r_{j}$, a due date $d_{j}$, a deadline $D_{j}$ and/or a weight $w_{j}$. All jobs have to be processed on a set of $m$ identical machines. Each machine can process only one job at a time. During the processing of the jobs, preemptions can be allowed or forbidden. In the first case, the processing of any job once started has to be completed without any interruptions. In the second case, the processing of any operation may be interrupted and resumed later, possibly on another machine. The objective is to construct a feasible schedule so as to minimize a given criterion. In this paper, we discuss several solution approaches for the problem considered and expose problems with an open complexity status. For the interested readers, we can recommend in addition the collections of results from [8] and [15]. Here, we do not consider approximate and online algorithms. We refer the interested reader e.g. to the papers [14] and [19].

This survey is organized as follows. In Section 2 we review most important results on problems without preemptions. Then we survey the results on preemptive problems

United Institute of Informatics Problems

Minsk, 220012 Belarus

E-mail: kravch@newman.bas-net.by

Fakultät für Mathematik, Otto-von-Guericke-Universität

Magdeburg, 39106 Germany

E-mail: frank.werner@mathematik.uni-magdeburg.de 
in Section 3. In Section 4 we present an overview, where we refer to the 2-digit and 3 -digit numberings of the particular results in the text.

\section{Problems without preemptions}

Whereas the overwhelming majority of scheduling problems appears to be NP-hard, problems with equal processing time jobs form a remarkable case which is still open for most problems. Intuitively, such problems look polynomially solvable.

Due to the possibility to enumerate the possible places of the jobs in an optimal schedule, the model reminds an assignment problem. Therefore, one can intuitively suppose the existence of a polynomial algorithm for any monotonic criterion. Nevertheless, since prime conflicts are caused by overlapping intervals most of the problems have an open complexity status.

A special case is the model with $p=1$. For most criteria, this model can be solved by a network flow algorithm. However, this approach cannot be applied to the general model with arbitrary $p$ since, in the case of $p=1$, the main conflicts among overlapping places for processing jobs disappear. We refer the reader to [3] for a survey.

\subsection{Classical criteria}

2.1.1 If the processing times can be arbitrary, problem $1\left|r_{j}, D_{j}\right|-$ is unary NP-hard by a polynomial reduction from the 3 -partition problem, see [30].

2.1.2 In [18], an $O(n \log n)$ algorithm has been proposed for problem $1 \mid r_{j}, p_{j}=p$, $D_{j}$, prec $\mid C_{\max }$. More precisely, the authors consider a problem with unit processing times and arbitrary rational release dates and deadlines. However, by an appropriate scaling one can show that these two models are equivalent. They show that it is possible to modify the release times and deadlines so as to reflect the partial order (i.e., for the problem considered the constraint prec is irrelevant) by assigning $r_{j}:=\max \left\{\left\{r_{j}\right\} \cup\left\{r_{i}+1 \mid T_{i} \prec T_{j}\right\}\right\}$ and $d_{j}:=\min \left\{\left\{d_{j}\right\} \cup\left\{d_{i}-1 \mid T_{j} \prec T_{i}\right\}\right\}$. After such an assignment condition, $T_{i} \prec T_{j}$ implies $r_{i}<r_{j}$ and $d_{i}<d_{j}$. By straightforward interchange arguments, one can show that any schedule for problem $1\left|r_{j}, p_{j}=p, D_{j}\right|$ $C_{\max }$ can be transformed into a schedule for problem $1 \mid r_{j}, p_{j}=p, D_{j}$, prec $\mid C_{\max }$ without changing the $C_{\max }$-value.

2.1.3 To solve problem $1\left|r_{j}, p_{j}=p, D_{j}\right| C_{\max }$, the concept of forbidden regions (intervals) has been proposed (see [18]), i.e., open intervals where no job can start. The set of all forbidden regions is formed iteratively. The main observation used is the following. Assume that one knows the set of forbidden regions in the interval $\left[r_{i}, D_{j}\right]$, and let $J_{1}, \ldots, J_{k}$ be the set of jobs with release times and deadlines from the interval $\left[r_{i}, D_{j}\right]$. Now we set $r_{1}=\ldots=r_{k}=r_{i}$ and $D_{1}=\ldots=D_{k}=D_{j}$ and find the largest value of $e$ such that all jobs $J_{1}, \ldots, J_{k}$ can be scheduled in $\left[e, D_{j}\right]$ under the condition that no job can start in a forbidden interval. Now, if $r_{i} \leq e<r_{i}+1$, then ]e-1, $r_{i}$ [ is declared as a forbidden region, since in any feasible schedule a job starting in $] e-1, r_{i}$ [ is not from $\left\{J_{1}, \ldots, J_{k}\right\}$ and hence, the set $\left\{J_{1}, \ldots, J_{k}\right\}$ is finished after $D_{j}$. After forming the set of all forbidden regions, the implementation of the earliest deadline scheduling rule gives an optimal schedule.

2.1.4 In [39], it has been shown that problem $P \mid p_{j}=1, D_{j}$, prec $\mid-$ is unary NP-hard by a polynomial reduction from the 3 -satisfiability problem. 
2.1.5 In [31], the authors have shown that even problem $P \mid p_{j}=1, D_{j}=3$, prec $\mid-$ is unary NP-hard. Note that problem $P \mid p_{j}=1, D_{j}=2$, prec $\mid$ - can be polynomially solved in $O(n)$ time.

2.1.6 The important question about the complexity status of problem $P 3 \mid p_{j}=$ 1, prec $\mid C_{\max }$ is still open whereas in [17], problem $P 2 \mid p_{j}=1$, prec $\mid C_{\max }$ was solved in $O\left(n^{3}\right)$ time by a reduction of the problem to the maximum matching problem.

2.1.7 In [7], an $O(n \log n)$ algorithm has been proposed for problem $P \mid r_{j}, p_{j}=$ p, outtree $\mid C_{\max }$ and it has been shown that problem $P \mid r_{j}, p_{j}=p$, intree $\mid C_{\max }$ is unary NP-hard.

2.1.8 In [34], a polynomial algorithm with complexity $O\left(n^{3} \log \log n\right)$ has been developed for problem $P\left|r_{j}, p_{j}=p, D_{j}\right|-$. The algorithm is based on an analysis of the structural properties of an optimal schedule. The main features of the optimal structure used in that paper are the following:

1. Any schedule is completely defined by the set of time slots $\left(1, t_{1}\right),\left(2, t_{2}\right), \ldots$, $\left(n, t_{n}\right)$, where $i=1, \ldots, n$ is the slot number and $t_{i}$ is the starting time of slot $i$.

2. If we know the set of all occupied time slots, then an optimal schedule can be constructed by the earliest deadline scheduling procedure.

3. If the earliest deadline scheduling procedure generates a sequence $\left(1, t_{1}\right), \ldots,\left(k, t_{k}\right)$, such that job $J_{l}$ processed in $\left(k, t_{k}\right)$ is late, then job $J_{l}$ cannot be scheduled in $\left(k, t_{k}\right)$ and has to be scheduled earlier. To provide this, one of the slots $\left(1, t_{1}\right), \ldots$, $\left(k-1, t_{k-1}\right)$ has to be pulled right. To this end, Simons chooses the closest to $\left(k, t_{k}\right)$ slot which is occupied by a job whose deadline exceeds $D_{l}$.

It has been shown that the use of these principles leads to the construction of an optimal schedule for problem $P\left|r_{j}, p_{j}=p, D_{j}\right|-$. The same algorithm solves the problems $P\left|r_{j}, p_{j}=p, D_{j}\right| C_{\max }$ and $P\left|r_{j}, p_{j}=p, D_{j}\right| \sum C_{j}$.

2.1.9 In [36], an $O\left(m n^{2}\right)$ time algorithm was proposed for problems $P\left|r_{j}, p_{j}=p, D_{j}\right|-$, $P\left|r_{j}, p_{j}=p, D_{j}\right| C_{\max }$, and $P\left|r_{j}, p_{j}=p, D_{j}\right| \sum C_{j}$. The algorithm is substantially based on the ideas from [18] and [34].

2.1.10 In [10], the following linear programming formulation was proposed for problem $P\left|r_{j}, p_{j}=p, D_{j}\right|-$ :

$$
\begin{gathered}
\sum_{i=1}^{z} x_{j i}=p, \quad j=1, \ldots, n \\
\sum_{j=1}^{n} x_{j, i+1}+\ldots+\sum_{j=1}^{n} x_{j, i+y} \leq m p, \quad i=0, \ldots, z-y \\
x_{j i}=0 \text { if } I_{i} \not \subset\left[r_{j}, D_{j}[, \quad i=1, \ldots, z, \quad j=1, \ldots, n\right. \\
0 \leq x_{j i} \leq p, \quad i=1, \ldots, z, \quad j=1, \ldots, n
\end{gathered}
$$

Here $x_{j i}$ is equal to the amount of job $J_{j}$ processed in the interval $I_{i}$, where $\left\{I_{i} \mid\right.$ $i \in\{1, \ldots, z\}\}=\left\{\left[r_{j}+k p, r_{j}+k p+p[\mid k \in\{\ldots,-1,0,1, \ldots\}\}\right.\right.$, and $y=\max \{k \mid$ $\left.I_{i+1} \cap \ldots \cap I_{i+k} \neq \emptyset, i \in\{0, \ldots, z-k\}\right\}$. In the above system, the polyhedron (1) and for each $i=0, \ldots, z-y$, with $i+e y \leq z$, the polyhedron (2) are integer. Nevertheless, their intersection is not an integer polyhedron and therefore, the obtained solution is not necessarily integer. Using an obtained solution, one can construct an optimal schedule in two equivalent ways, namely:

1. It is possible to find the intervals which are occupied in a feasible schedule. Then the earliest deadline scheduling procedure generates an optimal schedule. 
2. It is possible to transform the obtained solution into the form $x_{j i}^{*} \in\{0, p\}$. The obtained vector gives an optimal solution for problem $P\left|r_{j}, p_{j}=p, D_{j}\right|-$.

In both cases, the following property of an optimal solution holds: If $k=\min \left\{i \mid x_{j i}^{*} \neq\right.$ $0, j=1, \ldots, n\}$ for the optimal solution, then the time slot $I_{k}$ is occupied in an optimal schedule.

2.1.11 Problem $P\left|r_{j}, p_{j}=p, q_{j}\right| C_{\max }$ was considered in [40]. Here $q_{j}$ is the delivery time of job $J_{j}$. The delivery takes no machine time but after being processed on the machine, any job requires a delivery time for its completion. Thus, for any job $J_{j}$ the completion time $C_{j}$ equals $t_{j}^{s}+p+q_{j}$, where $t_{j}^{s}$ is the starting time of the processing of job $J_{j}$, whereas the machine on which job $J_{j}$ is scheduled processes this job in the time interval $\left[t_{j}^{s}, t_{j}^{s}+p\right.$ [. In [40], a pseudopolynomial algorithm with complexity $O\left(q_{\max } m n \log n+O(m k n)\right)$ was proposed for problem $P\left|r_{j}, p_{j}=p, q_{j}\right| C_{\max }$, where $k<n$ holds.

Note that problem $P\left|r_{j}, p_{j}=p, q_{j}\right| C_{\max }$ can be solved by the polynomial algorithm developed in [24] for problem $P\left|r_{j}, p_{j}=p, D_{j}\right| \max \varphi_{j}\left(C_{j}\right)$, where $\varphi_{j}\left(C_{j}\right)$ is the cost associated with job $J_{j}$ completed at time $C_{j}$.

Thus, to solve problem $P\left|r_{j}, p_{j}=p, q_{j}\right| C_{\max }$ it is sufficient to solve problem $P \mid$ $r_{j}, p_{j}=p, q_{j} \mid \max \left\{C_{j}+q_{j}\right\} \leq F$ for some $F$, where $F=D\left(I_{i}\right)$ for some $i \in\{1, \ldots, z\}$ and $D\left(I_{i}\right)$ is the right endpoint of interval $I_{i}$. ¿From [24], it follows that problem $P\left|r_{j}, p_{j}=p, q_{j}\right| \max \left\{C_{j}+q_{j}\right\} \leq F$ is equivalent to the following feasibility problem:

$$
\begin{gathered}
\sum_{i=1}^{z} x_{j i}=p, \quad j=1, \ldots, n \\
\sum_{j=1}^{n} x_{j, i+1}+\sum_{j=1}^{n} x_{j, i+2}+\ldots+\sum_{j=1}^{n} x_{j, i+y} \leq m p, \quad i=0, \ldots, z-y \\
x_{j i}=0 \text { if } R\left(I_{i}\right)<r_{j} \quad i=1, \ldots, z, \quad j=1, \ldots, n \\
x_{j i}=0 \text { if } D\left(I_{i}\right)+q_{j}>F \\
x_{j i} \geq 0, \quad i=1, \ldots, z, \quad j=1, \ldots, n
\end{gathered}
$$

Here $R\left(I_{i}\right)$ is the left endpoint of the interval $I_{i}$.

2.1.12 It has been shown in [10] that, to solve problem $P\left|r_{j}, p_{j}=p, D_{j}\right| \sum C_{j}$, it is sufficient to solve the following linear programming problem:

$$
\begin{aligned}
& \text { Minimize } \sum_{i=1}^{z} \sum_{j=1}^{n} D\left(I_{i}\right) x_{j i} \\
& \text { subject to (1), (2), (3), (4). }
\end{aligned}
$$

2.1.13 In [16], the proposed linear programming formulation for the above problem was transformed by means of the substitution $y_{t}=\sum_{s=1}^{t} \sum_{j=1}^{n} x_{j s}$ into the following form:

$$
\begin{gathered}
\text { Minimize } \sum_{i=1}^{z} D\left(I_{i}\right)\left(y_{i}-y_{i-1}\right) \\
\text { subject to } y_{z}-y_{0}=n \\
y_{i}-y_{i-1} \geq 0, \quad i=1, \ldots, n
\end{gathered}
$$




$$
\begin{gathered}
y_{i}-y_{i-y+1} \leq m, \quad i=y, \ldots, n \\
y_{0}=0 .
\end{gathered}
$$

The authors have shown that this model can be solved in $O\left(n^{4}\right)$ time.

2.1.14 To solve problem $P\left|r_{j}, p_{j}=p\right| \sum w_{j} C_{j}$, it is sufficient to solve the following linear program (see $[10])$ :

$$
\operatorname{Minimize} \sum_{i=1}^{z} \sum_{j=1}^{n} w_{j} D\left(I_{i}\right) x_{j i}
$$

subject to (1), (2), (3), (4).

Note that for problem $P\left|r_{j}, p_{j}=p\right| \sum w_{j} C_{j}$ as far as for the next problem $P \mid r_{j}, p_{j}=$ $p \mid \sum T_{j}$, we introduce a $D_{j}$-value for each job $J_{j}$ by setting $D_{j}=\max _{j}\left\{r_{j}\right\}+n p$.

In [11], it has been shown that, in order to solve problem $P\left|r_{j}, p_{j}=p\right| \sum T_{j}$, it is sufficient to

$$
\begin{gathered}
\text { Minimize } \sum_{i=1}^{z} \sum_{j=1}^{n} \max \left\{0, D\left(I_{i}\right)-d_{j}\right\} x_{j i} \\
\text { subject to (1), (2), (3), (4). }
\end{gathered}
$$

2.1.15 In [2], a polynomial time algorithm with the complexity $O\left(n^{6 m+1}\right)$ was proposed for problem $P m\left|r_{j}, p_{j}=p\right| \sum w_{j} U_{j}$. The algorithm is based on the following observations and definitions:

1. It is possible to restrict the set of starting times by $\left\{r_{j}+z p \mid z \in\{0, \ldots, n\}, j \in\right.$ $\{0, \ldots, n\}\}$.

2. If the set of time slots $\left(1, t_{1}\right), \ldots,\left(n, t_{n}\right)$ is known in advance for an optimal schedule, where $i=1, \ldots, n$ is a slot number and $t_{i}$ is the starting time of slot $i$, then the desired schedule can be constructed by the earliest due date rule.

3. The only situation when job $J_{j}$ follows $J_{i}$ with $d_{j}<d_{i}$ is the situation when $J_{i}$ is processed within $] r_{j}-p, r_{j}+p$ [, i.e., the starting time of $J_{i}$ is before $r_{j}$.

4. A profile is defined as a vector $\left(a_{1}, \ldots, a_{m}\right)$, where $a_{i} \in\left\{r_{j}+z p \mid z \in\{0, \ldots, n\}, j \in\right.$ $\{0, \ldots, n\}\}$, and $\max \left\{a_{1}, \ldots, a_{m}\right\}-\min \left\{a_{1}, \ldots, a_{m}\right\} \leq p$. In an optimal schedule, for any job $J_{j}$ scheduled at $t_{j}$, only a profile $a$ with $t_{j} \in\left\{a_{1}, \ldots, a_{m}\right\}$ can be prescribed.

5. $k[a, b]$ is defined as the set of early jobs from $\left\{J_{1}, \ldots, J_{k}\right\}$ scheduled between the profiles $a$ and $b$, and $W_{k}[a, b]$ is the maximal weight for such a set.

Now, dynamic programming can be applied by using the formulas

$$
\begin{gathered}
W_{k}[a, b]=\max \left\{W_{k}^{\prime}[a, b], W_{k-1}[a, b]\right\} \text { if } \max \left\{a_{1}, \ldots, a_{m}\right\}-p \leq r_{k}<\min \left\{b_{1}, \ldots, b_{m}\right\}, \\
\text { and } W_{k}[a, b]=W_{k-1}[a, b] \text { if } r_{k} \notin\left[\max \left\{a_{1}, \ldots, a_{m}\right\}-p, \min \left\{b_{1}, \ldots, b_{m}\right\}[,\right. \\
\text { where } W_{k}^{\prime}[a, b]=\max _{\substack{\min \left\{x_{1}, \ldots, x_{m}\right\} \in \in \\
\left[r_{k}, d_{k}-p\right]}}\left\{W_{k-1}[a, x]+w_{k}+W_{k-1}\left[x^{\prime}, b\right]\right\} .
\end{gathered}
$$

2.1.16 In [12], an $O\left(n^{5}\right)$ algorithm was proposed for problem $1\left|r_{j}, p_{j}=p\right| \sum U_{j}$. This algorithm is analogous to the algorithm from [2], however, the authors do not use $W_{k}[a, b]$ but $w_{k}[a, B]$, i.e., for the given $w, k$ and $a$, the authors minimize $B$. For the single machine case, $B$ is the length of the considered subschedule. Thus, they define: 
1. $k[a, \cdot]$ is the set of jobs from $\left\{J_{1}, \ldots, J_{k}\right\}$ such that $r_{j} \geq a$ holds,

2. $w_{k}[a, B]$ equals the minimal value $B$ such that it is possible to execute $w$ jobs from $k[a, \cdot]$ in the time interval $[a+p, b]$.

2.1.17 In [33], a polynomial time algorithm was proposed for problem $P \mid r_{j}, p_{j}=$ $p, D_{j} \mid L_{\max }$. It is based on a binary search technique and on the fact that the number of possible starting points is polynomial in the problem size.

2.1.18 In [13], the case with identical jobs and uniform parallel machines has been considered, i.e., when each machine has some given speed. It has been shown how to solve problems $Q\left|r_{j}, p_{j}=p\right| C_{\max }$ and $Q\left|r_{j}, p_{j}=p\right| \sum C_{j}$ in $O(n \log n)$ and $O\left(m n^{2 m+1}\right)$ time, respectively.

2.1.19 Note that currently the most interesting open problems are $P\left|r_{j}, p_{j}=p\right|$ $\sum U_{j}$ and $P\left|r_{j}, p_{j}=p, D_{j}\right| \sum w_{j} C_{j}$.

\subsection{Some generalizations}

2.2.1 In [6], a dynamic programming approach was used to solve polynomially problem $\operatorname{Pm}\left|r_{j}, p_{j}=p\right| \sum f_{j}$, where $\sum f_{j}$ is an objective function depending on the completion times $C_{j}$ such that $f_{j}$ is non-decreasing and $f_{i}-f_{k}$ is monotonic. Note that both classical criteria $\sum w_{j} C_{j}$ and $\sum T_{j}$ can be described in such a way.

2.2.2 In [13], the case with identical jobs and uniform parallel machines has been considered, i.e., when each machine has some given speed. It has been shown how to solve problems $Q\left|p_{j}=p\right| \sum \varphi_{j}$ and $Q\left|p_{j}=p\right| \max \varphi_{j}$ in $O\left(n^{2}\right)$ time, where $\max \varphi_{j}=$ $\max _{1 \leq j \leq n} \varphi_{j}\left(C_{j}\right)$, and $\varphi_{j}, j=1, \ldots, n$, are non-decreasing functions in the job completion times.

2.2.3 In [23], a linear programming approach was proposed for problem $P\left|r_{j}, p_{j}=p\right|$ $\sum f_{j}$, where $\sum f_{j}$ is the objective function from [6], i.e., $f_{j}$ depends on the completion time $C_{j}$ such that $f_{j}$ is non-decreasing and $f_{i}-f_{k}$ is monotonic.

The approach for problem $P\left|r_{j}, p_{j}=p\right| \sum f_{j}$ is analogous to that from [11] and consists in minimizing

$$
\begin{gathered}
\sum_{i=1}^{z} \sum_{j=1}^{n} f_{j}\left(D\left(I_{i}\right)\right) x_{j i} \\
\text { subject to }(1),(2), \\
x_{j i}=0 \text { if } R\left(I_{i}\right)<r_{j}, \quad i=1, \ldots, z, \quad j=1, \ldots, n \\
x_{j i} \geq 0, \quad i=1, \ldots, z, \quad j=1, \ldots, n .
\end{gathered}
$$

Remind that $R\left(I_{i}\right)$ is the left endpoint of the interval $I_{i}$.

2.2.4 In [24], a polynomial algorithm was developed for problem $P\left|r_{j}, p_{j}=p, D_{j}\right|$ $\max \varphi_{j}\left(C_{j}\right)$, where $\varphi_{j}\left(C_{j}\right)$ is any non-decreasing function associated with job $J_{j}$ completed at time $C_{j}$. The classical scheduling criteria belonging to $\max \varphi_{j}\left(C_{j}\right)$ are the minimization of maximum completion times $C_{\max }$, the minimization of maximum lateness $L_{\text {max }}=\max _{j}\left\{C_{j}-d_{j}\right\}$ and maximum tardiness $\max T_{j}=\max _{j}\left\{L_{j}, 0\right\}$. A polynomial algorithm for problem $P\left|r_{j}, p_{j}=p, D_{j}\right| \max \varphi_{j}\left(C_{j}\right)$ can be briefly described as follows:

Note that the number of intervals available for processing can be polynomially bounded. Therefore, the possible number of different values $\varphi_{j}\left(D\left(I_{i}\right)\right)$ is polynomially bounded, 
too. Take any $F=\varphi_{j}\left(D\left(I_{i}\right)\right)$ for some $i$ and $j$. Consider the following feasibility problem:

$$
\begin{gathered}
\sum_{i=1}^{z} x_{j i}=p, \quad j=1, \ldots, n \\
\sum_{j=1}^{n} x_{j, i+1}+\ldots+\sum_{j=1}^{n} x_{j, i+y} \leq m p, \quad i=0, \ldots, z-y \\
x_{j i}=0 \text { if } R\left(I_{i}\right)<r_{j} \quad i=1, \ldots, z, \quad j=1, \ldots, n \\
x_{j i}=0 \text { if } \varphi_{j}\left(D\left(I_{i}\right)\right)>F \\
x_{j i} \geq 0, \quad i=1, \ldots, z, \quad j=1, \ldots, n
\end{gathered}
$$

It is possible to find a solution for the above problem such that $x_{j, i} \in\{0, p\}$. At the same time, the obtained solution can be considered as a solution for problem $P$ | $r_{j}, p_{j}=p, D_{j} \mid \max \varphi_{j}\left(C_{j}\right) \leq F$. Applying the same procedure for all different values of $F=\varphi_{j}\left(D\left(I_{i}\right)\right)$, we can choose the minimal value of $F$. Since the number of different values $\varphi_{j}\left(D\left(I_{i}\right)\right)$ is polynomially bounded, the proposed algorithm is polynomial.

2.2.5 In [35], the following problem has been considered. With each of the $n$ jobs, there is associated a set of intervals. Each interval has a starting time and a finishing time. All data are assumed to be integers. The goal is to construct a feasible schedule so that each job is processed only in one of the prescribed intervals. This problem can be denoted as $P\left|\left[r_{j}^{k}, D_{j}^{k}\right], p_{j}=p\right|-$. It has been shown that the considered problem is unary $N P$-hard for the case of one machine and two prescribed intervals for each job by a polynomial reduction from the 3 -satisfiability problem.

2.2.6 In [22], the following problem has been considered. As before, for each job $J_{j}$, $j=1, \ldots, n$, a processing time $p_{j}=p$, a release date $r_{j}$, and a deadline $D_{j}$ are given. Besides we suppose that the time interval $\left[\min _{j}\left\{r_{j}\right\}, \max _{j}\left\{D_{j}\right\}\right.$ [ is divided into several intervals $\left[t_{1}, t_{2}\left[,\left[t_{2}, t_{3}\left[, \ldots,\left[t_{T-1}, t_{T}\left[\right.\right.\right.\right.\right.\right.$, where $\min _{j}\left\{r_{j}\right\}=t_{1} \leq t_{2} \leq \ldots \leq t_{T}=$ $\max _{j}\left\{D_{j}\right\}$, such that for each interval $\left[t_{g}, t_{g+1}\right.$ [ the number of available machines $m_{g+1}$ is known in advance. Note that we do not fix the concrete set of $m_{g+1}$ machines, i.e., at two different points of $\left[t_{g}, t_{g+1}\right.$ [, one can use different sets of $m_{g+1}$ machines. Preemption of processing is not allowed, i.e., the processing of any job started at time $t$ on one of the identical machines will be completed at time $t+p$ on the same machine. We want to find a feasible schedule such that the maximal number of machines used by $J_{1}, \ldots, J_{n}$ is minimal. The problem has been reduced to the following linear programming problem.

$$
\begin{gathered}
\text { Minimize } M \\
\text { subject to } \sum_{i=1}^{z} x_{j i}=p, \quad j \in\{1, \ldots, n\} \\
\sum_{j=1}^{n} x_{j, i+1}+\ldots+\sum_{j=1}^{n} x_{j, i+q} \leq \min \left\{M, m_{k+1}\right\} p, \\
\text { where } i \in\{0, \ldots, z-q\}, \quad q \in\{1, \ldots, y\}, \\
k \in\{1, \ldots, T-1\}, \\
I_{i+1} \cap \ldots \cap I_{i+q} \cap\left[t_{k}, t_{k+1}[\neq \emptyset\right. \\
x_{j i}=0 \text { if } R\left(I_{i}\right)<r_{j}, \quad i=1, \ldots, z, \quad j=1, \ldots, n
\end{gathered}
$$




$$
\begin{gathered}
x_{j i}=0 \text { if } D_{j}<D\left(I_{i}\right), \quad i=1, \ldots, z, \quad j=1, \ldots, n \\
x_{j i} \geq 0, \quad i=1, \ldots, z, \quad j=1, \ldots, n .
\end{gathered}
$$

If we set $x_{j i}$ equal to the amount of job $J_{j}$ processed in the interval $I_{i}$ and $M$ is the number of machines we want to minimize, then any feasible schedule for the scheduling problem under consideration can be described as a feasible solution of the above linear programming problem.

On the other hand, the solution $\left(x^{*}, M^{*}\right)$ obtained for the linear programming problem can be transformed into an optimal solution of the scheduling problem considered in polynomial time.

\section{Problems with preemptions}

Now we consider the following basic problem. There are $m$ parallel machines and $n$ jobs, each requiring an identical execution time $p$. With each job $J_{j}$, there is associated a release time $r_{j}$. The processing of any job may be interrupted arbitrarily often and resumed later on any machine. The objective is to construct a feasible schedule so as to minimize a given criterion.

3.1 It has been shown in [38] that problem $P \mid p_{j}=1$, prec, pmtn $\mid \sum C_{j}$ is unary NP-hard.

3.2 In [28], a technique based on linear programming has been developed. The proposed approach allows one to solve problem $R \mid r_{j}$,pmtn $\mid L_{\text {max }}$ in polynomial time.

3.3 Using a pseudopolynomial reduction from numerical matching with target sums, it has been proved that problem $P\left|p_{j}=p, p m t n\right| \sum w_{j} U_{j}$ is unary NP-hard [9].

3.4 It has been noted in [2] that the algorithm developed in [28] for problem $R$ | $r_{j}$,pmtn $\mid L_{\max }$ can be used to solve both problem $P \mid p_{j}=p$, pmtn $\mid \sum U_{j}$ and problem $Q \mid p_{j}=p$,pmtn $\mid \sum U_{j}$ in polynomial time.

3.5 Problem $P \mid r_{j}, p_{j}=p$,pmtn $\mid \sum U_{j}$ is unary NP-hard [20]. In fact, in [20] it was proved that problem $P\left|r_{j}, p_{j}=m, p m t n^{+}\right| \sum U_{j}$ is unary NP-hard, here $p m t n^{+}$means that preemptions can be made only at integer time points. However, the proposed NP-hardness proof also works for problem $P \mid r_{j}, p_{j}=p$, pmtn $\mid \sum U_{j}$.

3.6 It is interesting to note that problem $P|p m t n| \sum U_{j}$ is binary NP-hard [27], however, its complexity status under an unary encoding is still an open question.

3.7 Problem $P \mid r_{j}, p_{j}=p$, pmtn $\mid \sum C_{j}$ has been solved in [1]. It is possible to prove that for problem $P \mid r_{j}, p_{j}=p$, pmtn $\mid \sum C_{j}$, an optimal schedule can be found in the class of schedules, where each job $J_{j}$ is processed on machine $m$ only within an (possibly empty) interval $\left[S_{j, m}, C_{j, m}\left[\right.\right.$, such that $C_{j, m} \leq S_{j+1, m}$ for each $m$ and $j<n$, and $C_{j, m} \leq S_{j, m-1}$ for each $m>1$ and $j$.

Using such a structure of an optimal schedule, one can formulate the following linear program which will solve problem $P\left|r_{j}, p_{j}=p, p m t n\right| \sum C_{j}$ in polynomial time.

$$
\begin{gathered}
\text { Minimize } \quad \sum_{j=1}^{n} C_{j, 1} \\
\text { subject to } \quad S_{j, m} \geq r_{j}, \quad j=1, \ldots, n \\
\sum_{q=1}^{m}\left(C_{j, m}-S_{j, m}\right)=p, \quad j=1, \ldots, n
\end{gathered}
$$




$$
\begin{gathered}
S_{j, m}-C_{j, m} \leq 0, \quad j=1, \ldots, n, \quad m=1, \ldots, m \\
C_{j, q}-S_{j, q-1} \leq 0, \quad j=1, \ldots, n, \quad q=2, \ldots, m \\
C_{j, q}-S_{j+1, q} \leq 0, \quad j=1, \ldots, n-1, \quad q=1, \ldots, m
\end{gathered}
$$

3.8 Another idea was used in [25] for problem $Q \mid r_{j}, p_{j}=p$, pmtn $\mid \sum C_{j}$. Note that for problem $Q \mid r_{j}, p_{j}=p$, pmtn $\mid \sum C_{j}$, an optimal schedule can be found in the class of schedules, for which $C_{1} \leq C_{2} \leq \cdots \leq C_{n}$ holds, i.e., there exists an optimal solution in which the completion times are in the same order as the release times.

Let $s^{*}$ be an optimal schedule for problem $Q \mid r_{j}, p_{j}=p$,pmtn $\mid \sum C_{j}$ with $C_{1}\left(s^{*}\right) \leq \cdots \leq C_{n}\left(s^{*}\right)$. Further we set $r_{n+1}=r_{n}+n \cdot \max _{q}\left\{p / s_{q}\right\}$, i.e., $r_{n+1}$ is a time point after which no job will be processed. Each $C_{j}\left(s^{*}\right)$ belongs to some interval $\left[r_{i}, r_{i+1}\right]$. However, if we know for each $C_{j}\left(s^{*}\right)$ the corresponding interval $\left[r_{i}, r_{i+1}\right]$ such that $C_{j}\left(s^{*}\right) \in\left[r_{i}, r_{i+1}\right]$, then an optimal schedule can be easily found using a reduction to a network flow problem, see [29]. Thus, the main question is to know the interval $\left[r_{i}, r_{i+1}\right]$ for each $C_{j}\left(s^{*}\right)$ such that $C_{j}\left(s^{*}\right) \in\left[r_{i}, r_{i+1}\right]$. However, this difficulty can be avoided due to criterion $\sum C_{j}$. For any job $J_{j}$, let the time interval $\left[r_{i}, r_{i+1}\right]$ be such that $C_{j} \in\left[r_{i}, r_{i+1}\right]$. Taking into account that $r_{1}=0$, we obtain $C_{j}=\left(r_{2}-r_{1}\right)+$ $\left(r_{3}-r_{2}\right)+\cdots+\left(r_{i}-r_{i-1}\right)+\left(C_{j}-r_{i}\right)$. Due to this decomposition, we introduce the completion time of job $J_{j}$ for each interval $\left[r_{i}, r_{i+1}\right]$.

For each job $J_{j}$ with $j=1, \ldots, n$ and for each interval $\left[r_{i}, r_{i+1}\right]$ with $i=1, \ldots, n$, we define the value $C\left(J_{j}, r_{i}\right)$ such that $C\left(J_{j}, r_{i}\right)=C_{j}\left(s^{*}\right)$ if $\left.C_{j}\left(s^{*}\right) \in\right] r_{i}, r_{i+1}[$, but if $C_{j}\left(s^{*}\right) \leq r_{i}$, then we set $C\left(J_{j}, r_{i}\right)=r_{i}$, and if $C_{j}\left(s^{*}\right) \geq r_{i+1}$, then we set $C\left(J_{j}, r_{i}\right)=r_{i+1}$. So, for each $i=1, \ldots, n$, the values $r_{i} \leq C\left(J_{1}, r_{i}\right) \leq \cdots \leq C\left(J_{i}, r_{i}\right)$ $\leq C\left(J_{i+1}, r_{i}\right)=\cdots=C\left(J_{n}, r_{i}\right)=r_{i+1}$ define a partition of the interval $\left[r_{i}, r_{i+1}\right]$. In turn, each interval $\left[C\left(J_{j-1}, r_{i}\right), C\left(J_{j}, r_{i}\right)\right]$ is completely defined by the jobs processed in it. Thus, we denote by $v\left(J_{k}, M_{q}, J_{j}, r_{i}\right)$ the part (amount) of job $J_{k}$ processed in the interval $\left[C\left(J_{j-1}, r_{i}\right), C\left(J_{j}, r_{i}\right)\right]$ by machine $M_{q}$, i.e., the total processing time of job $J_{k}$ by machine $M_{q}$ in the interval $\left[C\left(J_{j-1}, r_{i}\right), C\left(J_{j}, r_{i}\right)\right]$ equals $\frac{v\left(J_{k}, M_{q}, J_{j}, r_{i}\right)}{s_{q}}$ and for any job $J_{k}$, equality $\sum_{q=1}^{m} \sum_{i=1}^{n} \sum_{j=1}^{n} v\left(J_{k}, M_{q}, J_{j}, r_{i}\right)=p$ holds. The values $C\left(J_{j}, r_{i}\right)$, where $j=1, \ldots, n, \quad i=1, \ldots, n$, and the values $v\left(J_{k}, M_{q}, J_{j}, r_{i}\right)$, where $i, j=1, \ldots, n, \quad k=j, \ldots, i, \quad q=1, \ldots, m$, define a feasible solution of the following linear program. For convenience, we introduce $C\left(J_{0}, r_{i}\right)=r_{i}$.

$$
\operatorname{Minimize} \sum_{i=1}^{n}\left(\left(C\left(J_{1}, r_{i}\right)-r_{i}\right)+\cdots+\left(C\left(J_{n}, r_{i}\right)-r_{i}\right)\right)
$$

subject to

$$
\begin{gathered}
r_{i}=C\left(J_{0}, r_{i}\right) \leq C\left(J_{1}, r_{i}\right) \leq \cdots \leq C\left(J_{i+1}, r_{i}\right)=\cdots=C\left(J_{n}, r_{i}\right)=r_{i+1}, \\
\sum_{q=1}^{m} \frac{v\left(J_{k}, M_{q}, J_{j}, r_{i}\right)}{s_{q}} \leq C\left(J_{j}, r_{i}\right)-C\left(J_{j-1}, r_{i}\right), \\
\sum_{k=1}^{n} \frac{v\left(J_{k}, M_{q}, J_{j}, r_{i}\right)}{s_{q}} \leq C\left(J_{j}, r_{i}\right)-C\left(J_{j-1}, r_{i}\right),
\end{gathered}
$$




$$
\sum_{i=1}^{n} \sum_{j=1}^{n} \sum_{q=1}^{m} v\left(J_{k}, M_{q}, J_{j}, r_{i}\right)=p
$$

where $i=1, \ldots, n, \quad j=1, \ldots, n, \quad k=1, \ldots, n, \quad q=1, \ldots, m$,

$$
C\left(J_{j}, r_{i}\right) \geq 0, \quad i=1, \ldots, n, \quad j=1, \ldots, n,
$$

and for $q=1, \ldots, m$

$$
\begin{gathered}
v\left(J_{k}, M_{q}, J_{j}, r_{i}\right)=0, \quad i=1, \ldots, n-1, \quad j=i+1, \ldots, n, \quad k=1, \ldots, n \\
v\left(J_{k}, M_{q}, J_{j}, r_{i}\right)=0, \quad i=1, \ldots, n, \quad j=1, \ldots, i, \quad k=1, \ldots, j-1, \\
v\left(J_{k}, M_{q}, J_{j}, r_{i}\right)=0, \quad i=1, \ldots, n-1, \quad j=1, \ldots, i, \quad k=i+1, \ldots, n, \\
v\left(J_{k}, M_{q}, J_{j}, r_{i}\right) \geq 0 \quad i=1, \ldots, n, \quad j=1, \ldots, n, \quad k=1, \ldots, n .
\end{gathered}
$$

The above formulation includes $O\left(m n^{3}\right)$ variables and constraints, i.e., this problem can be polynomially solved. In [25], it has been shown that an optimal solution of the above linear program can be used to obtain an optimal schedule.

3.9 Problem $P \mid r_{j}$, pmtn $\mid \sum C_{j}$ has been proved to be unary NP-hard by a reduction from 3-partition in [1].

3.10 Problem $P\left|r_{j}, p_{j}=p, p m t n\right| \sum w_{j} C_{j}$ is unary NP-hard [32].

3.11 In [21], it was shown that both the problem $P \mid$ pmtn $\mid \sum T_{j}$ of minimizing total tardiness on a set of parallel machines with allowed preemptions and problem $P\left|r_{j}, p_{j}=p, p m t n\right| \sum T_{j}$ of minimizing total tardiness on a set of parallel machines with release dates, equal processing times and allowed preemptions are NP-hard in the ordinary sense.

Moreover, in [21] problem $Q \mid p_{j}=p$, pmtn $\mid \sum f_{j}$ was considered, where $f_{j}$ are convex non-decreasing functions such that $f_{i}-f_{j}$ are all monotonic functions. It is shown that problem $Q \mid p_{j}=p$,pmtn $\mid \sum f_{j}$ is equivalent to the problem of minimizing a convex-separable function under linear constraints. This representation is used to solve problems $Q \mid p_{j}=p$,pmtn $\mid \sum T_{j}$ and $Q \mid p_{j}=p$, pmtn $\mid \sum w_{j} C_{j}$ in polynomial time.

3.12 Thus, the minimal open problems are $1\left|r_{j}, p_{j}=p, p m t n\right| \sum w_{j} C_{j}$ and $P \mid$ $r_{j}, p_{j}=p, p m t n \mid \sum T_{j}$ with respect to an unary encoding.

\section{Summary}

In Tables 1 and 2, we give an overview on the main results mentioned in this review. 
Table 1: Polynomially solvable and NP-hard problems: Non-preemptive problems

\begin{tabular}{|c|c|c|}
\hline $1\left|r_{j}, D_{j}\right|-$ & unary NP-hard [30] & 2.1 .1 \\
\hline$P \mid p_{j}=1, D_{j}$, prec $\mid-$ & unary NP-hard [39] & 2.1 .4 \\
\hline$P \mid p_{j}=1, D_{j}=3$, prec $\mid-$ & unary NP-hard [31] & 2.1 .5 \\
\hline$P \mid p_{j}=1, D_{j}=2$, prec $\mid-$ & solvable in $O(n)[31]$ & 2.1 .5 \\
\hline$P\left|r_{j}, p_{j}=p, D_{j}\right|-$ & solvable in $O\left(m n^{2}\right)[36]$ & $2.1 .8 \& 2.1 .9 \& 2.1 .10$ \\
\hline $1 \mid r_{j}, p_{j}=p, D_{j}$, prec $\mid C_{\max }$ & solvable in $O(n \log n)[18]$ & 2.1 .2 \\
\hline $1\left|r_{j}, p_{j}=p, D_{j}\right| C_{\max }$ & solvable in $O(n \log n)$ [18] & $2.1 .2 \& 2.1 .3$ \\
\hline$P 2 \mid p_{j}=1$, prec $\mid C_{\max }$ & solvable in $O\left(n^{3}\right)$ [17] & 2.1 .6 \\
\hline$P 3 \mid p_{j}=1$, prec $\mid C_{\max }$ & open & 2.1.6 \\
\hline$P \mid r_{j}, p_{j}=p$, outtree $\mid C_{\max }$ & solvable in $O(n \log n)[7]$ & 2.1 .7 \\
\hline$P \mid r_{j}, p_{j}=p$, intree $\mid C_{\max }$ & unary $N P$-hard $[7]$ & 2.1 .7 \\
\hline$P\left|r_{j}, p_{j}=p, D_{j}\right| C_{\max }$ & solvable in $O\left(m n^{2}\right)[36]$ & $2.1 .8 \& 2.1 .9$ \\
\hline$P\left|r_{j}, p_{j}=p, q_{j}\right| C_{\max }$ & reducible to $\mathrm{LP}$ & 2.1 .11 \\
\hline$Q\left|r_{j}, p_{j}=p\right| C_{\max }$ & solvable in $O(n \log n)$ [13] & 2.1 .18 \\
\hline$Q\left|p_{j}=p\right| C_{\max }$ & solvable in $O(n+m \log m)$ [13] & \\
\hline$Q\left|p_{j}=p\right| L_{\max }$ & solvable in $O(n \log n)$ [13] & \\
\hline$P\left|r_{j}, p_{j}=p, D_{j}\right| L_{\max }$ & solvable in $O\left(m n^{4}\right)$ [33] & 2.1 .17 \\
\hline$P\left|r_{j}, p_{j}=p, D_{j}\right| \sum C_{j}$ & solvable in $O\left(m n^{2}\right)[36]$ & $2.1 .8 \& 2.1 .9 \& 2.1 .12 \& 2.1 .13$ \\
\hline$Q\left|r_{j}, p_{j}=p\right| \sum C_{j}$ & solvable in $O\left(m n^{2 m+1}\right)$ [13] & 2.1 .18 \\
\hline$Q\left|p_{j}=p\right| \sum C_{j}$ & solvable in $O(n+m \log m)$ [13] & \\
\hline$P\left|r_{j}, p_{j}=p\right| \sum w_{j} C_{j}$ & reducible to LP [10] & 2.1 .14 \\
\hline$P\left|r_{j}, p_{j}=p, D_{j}\right| \sum w_{j} C_{j}$ & open & 2.1 .19 \\
\hline$Q\left|p_{j}=p\right| \sum w_{j} C_{j}$ & solvable in $O(n \log n)$ [13] & \\
\hline $1\left|r_{j}, p_{j}=p\right| \sum T_{j}$ & solvable in $O\left(n^{7}\right)[6]$ & \\
\hline$P\left|r_{j}, p_{j}=p\right| \sum T_{j}$ & reducible to LP [11] & 2.1 .14 \\
\hline$Q\left|p_{j}=p\right| \sum T_{j}$ & solvable in $O(n \log n)$ [13] & \\
\hline$Q\left|p_{j}=p\right| \max w_{j} T_{j}$ & solvable in $O\left(n \log ^{2} n\right)$ [13] & \\
\hline $1\left|r_{j}, p_{j}=p\right| \sum U_{j}$ & solvable in $O\left(n^{5}\right)[12]$ & 2.1 .16 \\
\hline$P\left|r_{j}, p_{j}=p\right| \sum U_{j}$ & open & 2.1 .19 \\
\hline $1\left|r_{j}, p_{j}=p\right| \sum w_{j} U_{j}$ & solvable in $O\left(n^{7}\right)[5]$ & \\
\hline $\operatorname{Pm}\left|r_{j}, p_{j}=p\right| \sum w_{j} U_{j}$ & solvable in $O\left(n^{6 m+1}\right)[2]$ & 2.1 .15 \\
\hline$Q\left|p_{j}=p\right| \sum w_{j} U_{j}$ & solvable in $O(n \log n)$ [13] & \\
\hline$P m\left|r_{j}, p_{j}=p\right| \sum f_{j}$ & solvable in $O\left(n^{3 m+4}\right)[6]$ & 2.2 .1 \\
\hline$Q\left|p_{j}=p\right| \sum \varphi_{j}$ & solvable in $O\left(n^{2}\right)[13]$ & 2.2 .2 \\
\hline$Q\left|p_{j}=p\right| \overline{\max } \varphi_{j}$ & solvable in $O\left(n^{2}\right)$ [13] & 2.2 .2 \\
\hline$P\left|r_{j}, p_{j}=p\right| \sum f_{j}$ & reducible to LP [23] & 2.2 .3 \\
\hline$P\left|r_{j}, p_{j}=p, D_{j}\right| \max \varphi_{j}$ & reducible to LP [24] & 2.2 .4 \\
\hline$P\left|\left[r_{j}^{k}, D_{j}^{k}\right], p_{j}=p\right|-$ & unary NP-hard [35] & 2.2 .5 \\
\hline$P\left|r_{j}, p_{j}=p, D_{j}\right| m$ & reducible to LP [22] & 2.2 .6 \\
\hline
\end{tabular}


Table 2: Polynomially solvable and NP-hard problems: Preemptive problems

\begin{tabular}{|c|c|c|}
\hline$P \mid p_{j}=1$, prec, pmtn $\mid \sum C_{j}$ & unary NP-hard [38] & 3.1 \\
\hline$P \mid r_{j}$, pmtn $\mid \sum C_{j}$ & unary NP-hard [1] & 3.9 \\
\hline$P\left|r_{j}, p_{j}=p, p m t n\right| \sum C_{j}$ & reducible to LP [1] & 3.7 \\
\hline$Q\left|r_{j}, p_{j}=p, p m t n\right| \sum C_{j}$ & reducible to LP [25] & 3.8 \\
\hline $1\left|r_{j}, p_{j}=p, p m t n\right| \sum w_{j} C_{j}$ & open (unary encoding) & 3.12 \\
\hline$P\left|r_{j}, p_{j}=p, p m t n\right| \sum w_{j} C_{j}$ & unary NP-hard [32] & 3.10 \\
\hline$Q\left|p_{j}=p, p m t n\right| \sum w_{j} C_{j}$ & reducible to LP [21] & \\
\hline$R \mid r_{j}$, pmtn $\mid L_{\max }$ & reducible to LP [28] & 3.2 \\
\hline $1\left|r_{j}, p_{j}=p, p m t n\right| \sum U_{j}$ & solvable in $O(n \log n)[26]$ & \\
\hline$P\left|r_{j}, p_{j}=p, p m t n\right| \bar{\sum} U_{j}$ & unary NP-hard [20] & 3.5 \\
\hline$P \mid$ pmtn $\mid \sum U_{j}$ & binary NP-hard [27] & 3.6 \\
\hline$Q\left|p_{j}=p, p m t n\right| \sum U_{j}$ & solvable in $O\left(n \log ^{2} n+m n \log n\right)$ [2] & 3.4 \\
\hline $1\left|r_{j}, p_{j}=p, p m t n\right| \sum w_{j} U_{j}$ & solvable in $O\left(n^{4}\right)[4]$ & \\
\hline$P\left|p_{j}=p, p m t n\right| \sum \bar{w}_{j} U_{j}$ & unary NP-hard [9] & 3.3 \\
\hline $1\left|r_{j}, p_{j}=p, p m t n\right| \sum T_{j}$ & solvable in $O\left(n^{2}\right)[37]$ & \\
\hline$P\left|r_{j}, p_{j}=p, p m t n\right| \sum T_{j}$ & binary NP-hard [21] & 3.11 \\
\hline$P\left|r_{j}, p_{j}=p, p m t n\right| \sum T_{j}$ & open (unary encoding) & 3.12 \\
\hline$P|p m t n| \sum T_{j}$ & binary NP-hard [21] & 3.11 \\
\hline$Q\left|p_{j}=p, p m t n\right| \sum T_{j}$ & reducible to LP [21] & 3.11 \\
\hline$Q\left|p_{j}=p, p m t n\right| \sum f_{j}$ & reducible to LP [21] & 3.11 \\
\hline
\end{tabular}

Acknowledgements This work was partially supported by the Alexander von Humboldt Foundation.

\section{References}

1. P. Baptiste, P. Brucker, M. Chrobak, C. Dürr, S.A. Kravchenko, and F. Sourd, The complexity of mean flow time scheduling problems with release times, J. Scheduling, 10, 139-146, 2007.

2. P. Baptiste, P. Brucker, S. Knust, and V. G. Timkovsky, Ten notes on equal-processing-time scheduling, 4OR, 2, 111-127, 2004.

3. P. Baptiste, and P. Brucker, Scheduling equal processing time jobs: a survey, In Y.T. Leung, Handbook of Scheduling: Algorithms, Models, and Performance Analysis, 78-96, CRC Press LLC, Boca Raton, FR, 2004.

4. P. Baptiste, M. Chrobak, C. Dürr, W. Jawor, and N. Vakhania, Preemptive scheduling of equal-length jobs to maximize weighted throughput, Oper. Res. Letters, 32, 258-264, 2004.

5. P. Baptiste, Polynomial time algorithms for minimizing the weighted number of late jobs on a single machine with equal processing times, J. of Scheduling, 2 (6), 245-252, 1999.

6. P. Baptiste, Scheduling equal-length jobs on identical parallel machines, Discrete Appl. Math., 103, 21-32, 2000.

7. P. Brucker, M. R. Garey, and D. S. Johnson, Scheduling equal-length tasks under tree-like precedence constraints to minimize maximum lateness, Math. Oper. Res., 2, 275-284, 1977.

8. P. Brucker, S. Knust, Complexity results for scheduling problems, http://www.mathematik.uni-osnabrueck.de/research/OR/class/

9. P. Brucker, S.A. Kravchenko, Scheduling equal processing time jobs to minimize the weighted number of late jobs, Journal of Mathematical Modelling and Algorithms, 5(2), 143-165, 2006.

10. P. Brucker, S.A. Kravchenko, Scheduling jobs with equal processing times and time windows on identical parallel machines, J. Scheduling, 11, 229-237, 2008.

11. P. Brucker, S.A. Kravchenko, Scheduling jobs with release times on parallel machines to minimize total tardiness, Universität Osnabrück, Preprint Heft 258, 13 pp., 2005. 
12. M. Chrobak, C. Dürr, W. Jawor, Ł. Kowalik, and M. Kurowski, A note on scheduling equal-length jobs to maximize throughput, J. Scheduling, 9, 71-73, 2006.

13. M.I. Dessouky, B.J. Lageweg, J.K. Lenstra, and S.L. van de Velde, Scheduling identical jobs on uniform parallel machines, Statistica Neerlandica, 44, 115-123, 1990.

14. J. Ding, T. Ebenlendr, J. Sgall, G. Zhang, Online-scheduling of equal-length jobs on parallel machines, Lect. Notes Comput. Sci., 4698, 427 - 438, 2007.

15. C. Dürr, The scheduling zoo, http://www.lix.polytechnique.fr/ durr/query/

16. C. Dürr, M. Hurrand, Finding total unimodularity in optimization problems solved by linear programs, Proc. of the 14th Annual European Symposium on Algorithms (ESA), 315$326,2006$.

17. M. Fujii, T. Kasami, and K. Ninomiya, Optimal sequencing of two equivalent processors, SIAM J. Appl. Math., 17, 234-248, 1969.

18. M.R. Garey, D.S. Johnson, B.B. Simons, and R.E. Tarjan, Scheduling unit-time tasks with arbitrary release times and deadlines, J. Comput., 10, 256-269, 1981.

19. Y. Huo, J.Y-T. Leung, X. Wang, Online scheduling of equal-processing-time task systems, Theor. Comput. Sci., 401, 85 - 95, 2008.

20. S.A. Kravchenko, On the complexity of minimizing the number of late jobs in unit time open shop, Discrete Appl. Math., 100, 127-132, 2000.

21. S.A. Kravchenko, F. Werner, Minimizing a separable convex function on parallel machines with preemptions, Otto-von-Guericke-Universität Magdeburg, FMA, Preprint 22/09, 20 pp., 2009 .

22. S.A. Kravchenko, F. Werner, Minimizing the number of machines for scheduling jobs with equal processing times, European J. Oper. Res., 199, 595-600, 2009.

23. S.A. Kravchenko, F. Werner, On a parallel machine scheduling problem with equal processing times, Discrete Appl. Math., 157, 848-852, 2009.

24. S.A. Kravchenko, F. Werner, On a parallel machine scheduling problem with equal processing times, Otto-von-Guericke-Universität Magdeburg, FMA, Preprint 26/07, 9 pp., 2007.

25. S.A. Kravchenko, F. Werner, Preemptive scheduling on uniform machines to minimize mean flow time, Comput. Oper. Res., Vol. 36(10), 2816-2821, 2009.

26. E.L. Lawler, Knapsack-like scheduling problems, the Moore-Hodgson algorithm and the 'tower of set' property, Math. Comput. Modelling, 20(2), 91-106, 1994.

27. E.L. Lawler, Recent results in the theory of machine scheduling, In A. Bachem, Mathematical Programming: the state of the Art, 202-234, Springer, Berlin, 1982.

28. E. L. Lawler, J. Labetoulle, On preemptive scheduling of unrelated parallel processors by linear programming, J. Assoc. Comput. Mach., 25(4), 612-619, 1978.

29. J. Labetuolle, E.L. Lawler, J.K. Lenstra, and A.H.G. Rinnooy Kan, Preemptive scheduling of uniform machines subject to release dates, in H.R. Pulleyblank, Progress in Combinatorial Optimization, 245-261, Academic Press, New York, 1984.

30. J.K. Lenstra, A.G.H. Rinnooy Kan, and P. Brucker, Complexity of machine scheduling problems, Ann. Discrete Math., 1, 343-362, 1977.

31. J.K. Lenstra, A.H.G. Rinnooy Kan, Complexity of scheduling under precedence constraints, Oper. Res., 26, 22-35, 1978.

32. J.Y.-T. Leung, G.H. Young, Preemptive scheduling to minimize mean weighted flow time, Information Processing Letters, 34, 47-50, 1990.

33. B. Simons, A fast algorithm for single processor scheduling, Proc. IEEE 19th Annual Symposium on Foundations of Computer Science (FOCS'r8), 246-252, 1978.

34. B. Simons, Multiprocessor scheduling of unit-time jobs with arbitraty release times and deadlines, SIAM J. Comput., 12, 7-9, 1983.

35. B.B. Simons, M. Sipser, On scheduling unit-length jobs with multiple release time/deadline intervals, Oper. Res., 32, 80-88, 1984.

36. B.B. Simons, M.K. Warmuth, A fast algorithm for multiprocessor scheduling of unit-length jobs, SIAM J. Comput., 18, 690-710, 1989.

37. Z. Tian, C.T. Ng, T.C.E. Cheng, An $O\left(n^{2}\right)$ algorithm for scheduling equal-length preemptive jobs on a single machines to minimize total tardiness, J. Scheduling, 9, 343-364, 2006.

38. J.D. Ullman, Complexity of sequencing problems, in J.L. Bruno, E.G. Coffman, Jr., R.L. Graham, W.H. Kohler, R. Sethi, K. Steiglitz, and J.D. Ullman, Computer and Job/Shop Scheduling Theory, John Wiley \& Sons Inc., New York, 1976.

39. J.D. Ullman, NP-complete scheduling problems, J. Comput. System Sci., 10, 384-393, 1975.

40. N. Vakhania, A better algorithm for sequencing with release and delivery times on identical machines, J. Algorithms, 48, 273-293, 2003. 